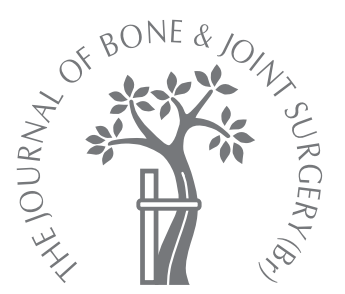

\title{
Observations on the natural history of massive lumbar disc herniation
}

\author{
G. L. Cribb, \\ D. C. Jaffray, \\ V. N. Cassar- \\ Pullicino
}

From the Robert

Jones and Agnes

Hunt Orthopaedic

Hospital, Oswestry,

England
G. L. Cribb, FRCS(Tr \& Orth),

Specialist Registrar

D. C. Jaffray, FRCS,

Consultant Orthopaedic

Surgeon

V. N. Cassar-Pullicino, FRCR,

Consultant Musculoskeletal

Radiologist

Robert Jones and Agnes Hunt

Orthopaedic Hospital,

Oswestry, Shropshire SY10

7AG, UK.

Correspondence should be sent to Miss G. L. Cribb; e-mail: gcribb@hotmail.com

(C2007 British Editorial Society of Bone and Joint Surgery doi:10.1302/0301-620X.89B6 $18712 \$ 2.00$

$J$ Bone Joint Surg $[B r]$ 2007;89-B:782-4.

Received 28 September 2006; Accepted after revision 2 March 2007

\author{
We have treated 15 patients with massive lumbar disc herniations non-operatively. Repeat \\ MR scanning after a mean 24 months (5 to 56) showed a dramatic resolution of the \\ herniation in 14 patients. No patient developed a cauda equina syndrome. \\ We suggest that this condition may be more benign than previously thought.
}

Most herniated lumbar discs resolve spontaneously. ${ }^{1-8}$ Although smaller herniations may be safely treated non-operatively, massive extrusions and sequestrations are sometimes treated by operation for fear of cauda equina compression. We present the clinical and radiological outcome of 15 patients who were treated conservatively having presented with leg pain and a massive lumbar disc herniation.

\section{Patients and Methods}

We have reviewed 15 patients with a massive lumbar disc herniation and a painful radiculopathy who chose not to have surgery. They presented to a general spinal clinic over a period of five years. There were ten women and five men, with a mean age of 45 years ( 24 to 73). Ten herniations were at the L4-5 level and five at L5-S1. The reason the patients chose to be treated without operation was either that their symptoms had started to improve spontaneously, that they feared surgical complications, or both. To qualify as a 'massive' herniation at least $50 \%$ of the spinal canal had to be occluded by disc material on axial MRI scans. The anteroposterior diameters of the spinal canal and of the disc were measured and the latter expressed as a percentage of the former. The patients were advised to return for emergency discectomy should they develop features of a cauda equina syndrome. Otherwise they were asked back for clinical review and a repeat MR scan. These scans were performed at a mean of 24 months (5 to 56) after the initial scan. All were performed without gadolinium enhancement.

The following criteria were used to distinguish protrusion from extrusion. The disc herniation was deemed to be a protrusion if the greatest distance, in any plane, between the edges of the disc material beyond the disc space was less than the distance between the edges of the base in the same plane. A disc extrusion was deemed to be present if any one distance between the edges of the disc material beyond the disc space was greater than the distance between the edges of the base measured in the same plane. An extrusion was designated a sequestration if the displaced disc material had completely lost continuity with the parent disc. $^{9}$

\section{Results}

All 15 disc herniations were classified as extrusions, six of which were sequestrations. All were uncontained as there was no surrounding annulus.

The mean percentage of the canal occupied by disc on an axial MR scan was $66 \%$ (55\% to $80 \%)$. All but one herniation had resolved dramatically by the time of the second MR scan (Fig. 1). The reduction in size of the herniation on MRI was a mean of $80 \%(68 \%$ to $100 \%$ ).

One patient needed a discectomy because of persistent pain, despite substantial resolution of the disc prolapse on MRI. In another patient whose disc had not resolved radiologically, the symptoms had diminished to such an extent that surgery was not required.

No patient developed a cauda equina syndrome.

\section{Discussion}

In 1983 , Weber ${ }^{1}$ showed that the natural history of radiculopathy because of lumbar disc herniation is to clinical resolution. Surgery carried out in the first year gave earlier relief of pain, but thereafter the results of surgery were the same as those of non-operative treatment. 

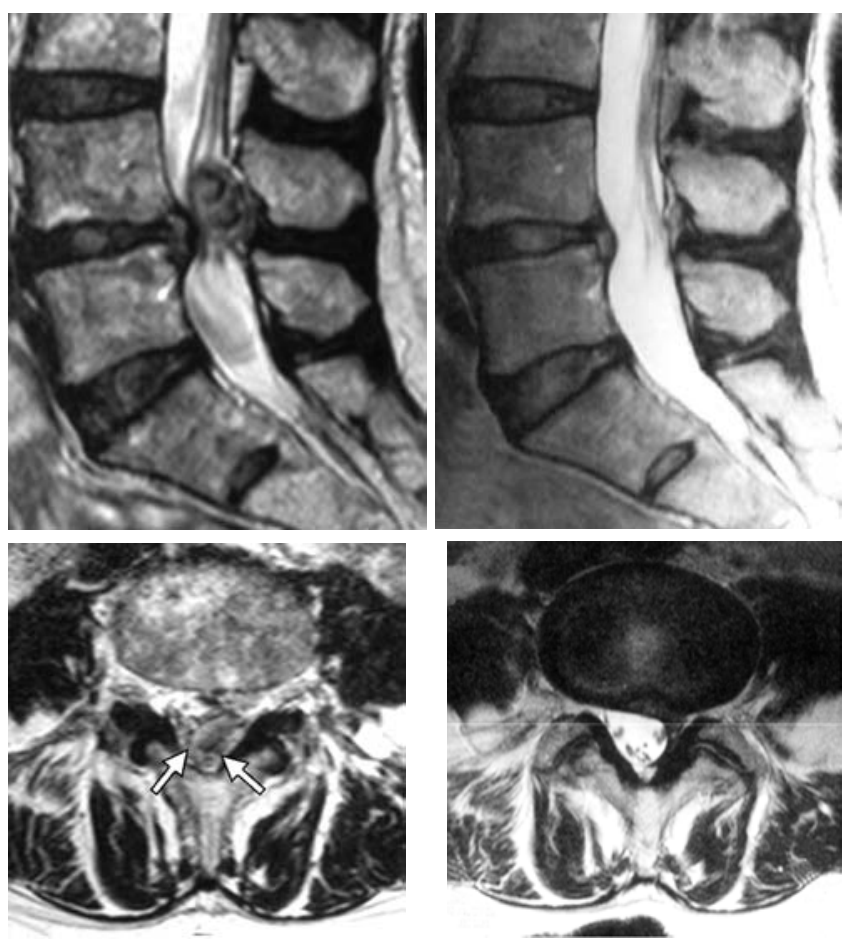

Fig. 1

Resolution of a massive extrusion with disc sequestration after six months.

Weber's study predates MR scanning, and it is understandable that there was no myelographic follow-up to see the radiological outcome of non-operative treatment. There may well have been massive herniations in that series, but it is likely that they would have formed a tiny proportion of the overall number, just as we find in our practice.

The spontaneous resolution of lumbar disc herniation has been demonstrated both on CT scanning $2,5,6$ and clinically. ${ }^{3,10}$ Jensen et $\mathrm{al}^{8}$ studied the changes in disc morphology in 154 patients with sciatica who were treated non-operatively. They found that broad-based protrusions, extrusions and sequestrations improved more than bulges and focal protrusions. They concluded that nerve root compromise had the best prognosis if the disc was extruded on the baseline MR scan. Porter, Hibbert and Wicks ${ }^{11}$ examined the size of the spinal canal and showed that patients with small canals at the L5 level were more likely to require discectomy. There was no relationship between the type of herniation, protrusion or sequestration and canal diameter in those who underwent surgery. The authors did not examine the size of the disc herniations. In our study we examined the size of the herniation in relation to the diameter of the canal. It would be interesting to extend this and compare canal size in our cohort of patients to a matched group of patients with massive disc herniations who required surgery.
There are two studies ${ }^{7,10}$ which have shown that larger disc herniations decrease in size and to a greater extent than do smaller protrusions.

Bozzao et $\mathrm{al}^{7}$ had eight patients in their series of 69 with disc herniations which occupied more than $50 \%$ of the canal. Six of these had reduced in size by more than $70 \%$ on follow-up axial MR scans.

The dramatic resolution of these massive extrusions is probably due to the loss of the immune privilege that the normal disc enjoys when covered by the outer annulus. Only when the disc ruptures are macrophages in the epidural space free to act on the disc material. Nevertheless, the ability of the body to clear the spinal canal of a massive extrusion is impressive.

The fear of a missed cauda equina syndrome prompts some surgeons to operate on massive discs. This fear may be misplaced. In our admittedly limited study, treating massive extrusions non-operatively did not result in complications. Surgery for lumbar disc herniation, by contrast, is not uncomplicated. The rate of recurrence in our institution is $7.9 \%{ }^{12}$ requiring further and more difficult surgery through dense scar tissue surrounding the affected nerve root. An audit of discectomies by the British Association of Spine Surgeons ${ }^{13}$ revealed a leak of cerebrospinal fluid in $3.5 \%$ of primary discectomies and $13.2 \%$ with revision discectomy. Infection rates of up to $3 \%$ have been reported, ${ }^{14}$ and death or serious injury through intra-abdominal vascular or visceral perforation has been described in 21 cases by Goodkin and Laska. ${ }^{15}$

Although massive herniations are rarely left alone for well-understood reasons, this small cohort of patients reveals a more benign side to this pathology which may not always deserve its fearsome reputation.

No benefits in any form have been received or will be received from a commercial party related directly or indirectly to the subject of this article.

\section{References}

1. Weber H. Lumbar disc herniation: a controlled, prospective study with ten years of observation. Spine 1983;8:131-40.

2. Bush K, Cowan N, Katz DE, Gishen P. The natural history of sciatica associated with disc pathology: a prospective study with clinical and independent radiological follow-up. Spine 1992;17:1205-12.

3. Saal JA, Saal JS. Nonoperative treatment of herniated lumbar intervertebral disc with radiculopathy: an outcome study. Spine 1989;4:431-7.

4. Awad J, Moskovich R. Lumbar disc herniations: surgical versus nonsurgical treatment. Clin Orthop 2006;443:183-97.

5. Fagerlund MKJ, Thelander U, Friberg S. Size of lumbar disc hernias measured using computed tomography and related to sciatic symptoms. Acta Radiol 1990;31:555-8.

6. Teplick JG, Haskin ME. Spontaneous regression of herniated nucleus pulposus. AJR Am J Roentgenol 1985;6:371-5

7. Bozzao A, Gallucci M, Masciocchi C. Lumbar disc herniation: MR imaging assessment of natural history in patients treated without surgery. Radiology 1992:185:135-41.

8. Jensen TS, Albert HB, Soerensen JS, Manniche C, Leboeuf-Yde C. Natural course of disc morphology in patients with sciatica: an MRI study using a standardized qualitative classification system. Spine 2006;31:1605-12.

9. Fardon DF, Milette PC. Nomenclature and classification of lumbar disc pathology. Spine 2001;5:93-113.

10. Saal JA, Saal JS, Herzog RJ. The natural history of lumbar intervertebral disc extrusions treated nonoperatively. Spine 1990;15:683-6. 
11. Porter RW, Hibbert CS, Wicks $\mathbf{M}$. The spinal canal in symptomatic lumbar disc lesions. J Bone Joint Surg [Br] 1978;60-B:485-7.

12. Morgan-Hough CV, Jones PW, Eisenstein SM. Primary and revision lumbar discectomy: a 16-year review from one centre. J Bone Joint Surg [Br] 2003;83-B:871-4.

13. Tafazal SI, Sell PJ. Incidental durotomy in lumbar spine surgery: incidence and management. Eur Spine J 2005;14:287-90.
14. Rohde V, Meyer B, Schaller C, Hassler WE. Spondylodiscitis after lumbar discectomy: incidence and a proposal for prophylaxis. Spine 1998;23:615-20.

15. Goodkin R, Laska LL. Vascular and visceral injuries associated with lumbar disc surgery: medicolegal implications. Surg Neurol 1998;49:358-72. 